\title{
Electromyographic Studies on Urinary Tract
}

I. Fundamental Studies on EMG of Animal and Human Ureters

By

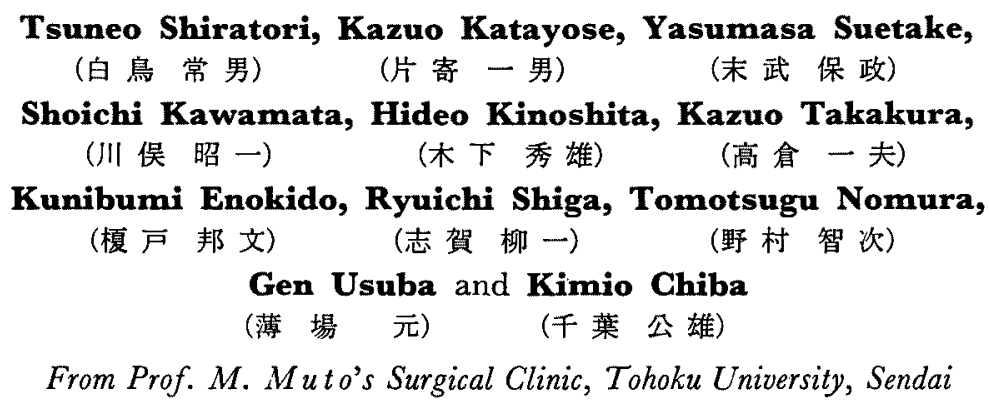

(Received for publication, December 18, 1958)

\section{INTRODUCTION}

Since the investigation by Orbeli and Brücke ${ }^{1)}$ on the action potential of the ureter many works have been reported by Hasama ${ }^{21}$, Aiba ${ }^{33}$, Bozler $^{4)}$, $\mathrm{Yano}^{5)}, \mathrm{Niu}^{6 / 7)}$, Obama ${ }^{8)}, \mathrm{Kudo}^{9)}$ and others, but all these investigations refer to animal ureters, and there are as yet many points of contention concerning the wave patterns observed in these studies. Recently more advanced research methods have become available, so that the results obtained by Sleator ${ }^{10)}$, Butcher ${ }^{11}$ and others, using these advanced methods are more reliable, and thus the research on this subject has come to turn a new page.

The present authors have been interested in this subject and made some observations on the human ureters in comparison with animal ureters; in the present investigation patients with apparently normal urinary organs subjected to laparotomy for intestinal diseases were chosen as subjects, and action potentials of their exposed ureters were recorded over a sufficiently long period. The nature of the wave patterns obtained has been critically studied. The result will be reported in the following.

\section{EXPERIMENTAL}

Methods of Observation and Experiment

1) As experimental animals, 42 adult dogs $(6-13 \mathrm{~kg}$.) were used. They were fixed in dorsal position in a laboratory $10-25^{\circ} \mathrm{C}$ in temperature and laparotomized under anesthesia with thiopental-sodium administerated 
intravenously in a dosis of $10 \mathrm{mg} / \mathrm{kg}$. Then the retroperitoneum was opened, the ureters were bluntly, detached for 2-4 cm. upwards, from a point about $4-8 \mathrm{~cm}$. above their attachment to the bladder, and action potentials were led off from these exposed parts of the ureters.

2) The human subjects were 12 patients of intestinal diseases clinically diagnosed as having intact urinary organs. After laparotomy their ureters were detached from the retroperitoneum to a length of about $4 \mathrm{~cm}$., in a region somewhat proximal to the line a terminalis, and action potentials were recorded from these exposed parts.

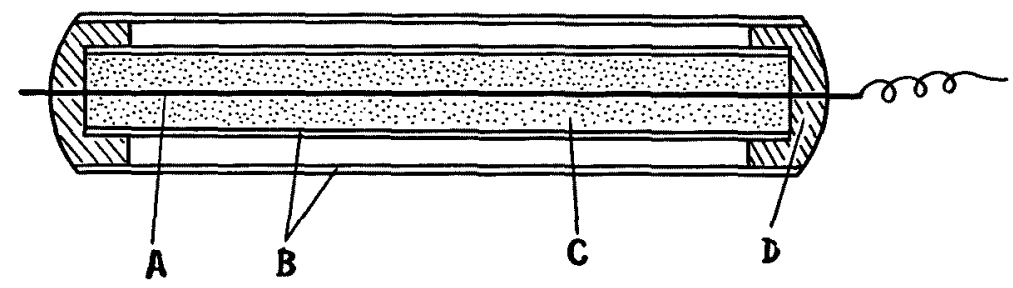

Fig. 1. A is a silver or iron wire $0.05-0.1 \mathrm{~mm}$. in diameter. $B$, glass pipettes, these cover A. C, (paraffin) D, (sealing wax)

For recording action potentials a four stage amplifier (one CR-coupled and three direct) by San-ei \& Co. or the Poly-Viso by Sanborn \& Co. was used. A silver wire $0.05-0.1 \mathrm{~mm}$. in diameter covered with a glass pipette except at the tip and fixed with paraffin, served as a different electrode. This electrode was made following a suggestion from Dr. Niu, Kyoto University (See Fig. 1). In some cases an iron wire of the same diameter was used instead of the silver wire. The electrode was introduced into the muscle layer of the ureter through its adventitia and kept suspended so that it was movable in accordance with the peristaltic movement of the ureter.

For unipolar leading, the different electrode was placed in the ureter, and the indifferent electrode of similar construction in the fatty tissue around the ureter. For bipolar leading one of the electrodes was placed at the proximal and the other at the distal end of the exposed ureter.

The recording instrument used was, the heat-writing recorder attached to the Poly-Viso or the H-type vibrator of Yokokawa's electromagnetic oscillograph. In records, the full length of a vertical bar represents $1 \mathrm{mV}$ and that of a horizontal bar 1 or 10 seconds.

\section{Experimental Results}

Wave patterns. As shown in Fig. 2A (dog) and 2B (man), fourphasic action potentials appeared one after another with certain regular intervals corresponding with peristalsis. Action potentials of man and dog are quite similar in wave-form. Upon recording these waves at a 

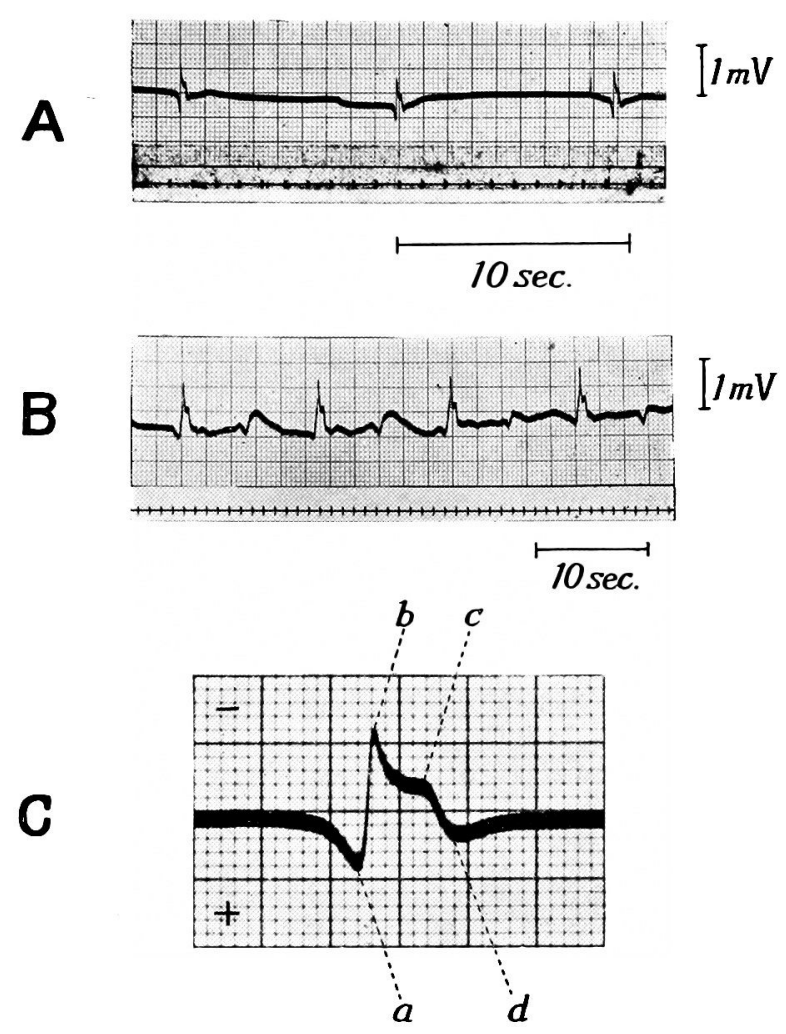

Fig. 2. A shows EUG in normal dogs. B shows EUG in human subjects. $\mathrm{C}$ is a typical wave pattern in $\mathrm{dog}$ or man. $a$, positive preceding deflectio.. $b$, major negative deflection. $c$, minor negative deflection. $d$, positive after potential.

higher velocity, we found, as shown in Fig. 2C, that a conspicuous major negative deflection and an accompanying minor negative deflection came between a positive preceding deflection and another positive after-potential.

Besides such a typical wave pattern, some cases, human or canine, were found to show potentials in which the minor negative deflection, and both positive deflections were less prominent. The wave patterns could be classified into the 3 following types (Figs. 3 and 4). Besides the typical wave pattern mentioned above (Type I), we found cases with indistinct minor negative deflection (Type II) and cases in which the principal negative deflection alone was remarkable, but both positive deflections were lacking (Type III). Fig. 3 shows potentials recorded with the silver electrodes, the amplifier having a time constant of $10 \mathrm{sec}$. and the Yokokawa's oscillograph while Fig. 4 shows those recorded with the iron electrodes, Poly-Viso having a time constant of $2.5 \mathrm{sec}$. and the heat-writing 


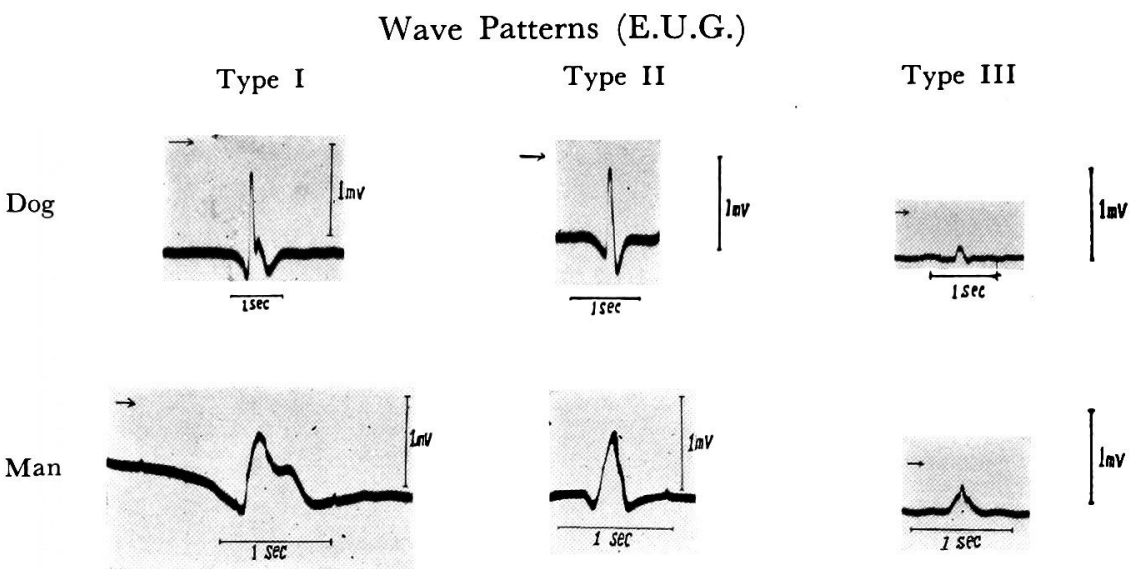

Fig. 3. For recording action potentials a four stage amplifier (one CRcoupled and three direct, time constant : 10 sec.) by San-ei \& Co., the silver electrodes and the Yokokawa's oscillograph were used. Type I, typical wave pattern; Type II, with indistinct minor negative deflection; Type III, principal negative deflection alone. The arrow-marks show the direction of progress.

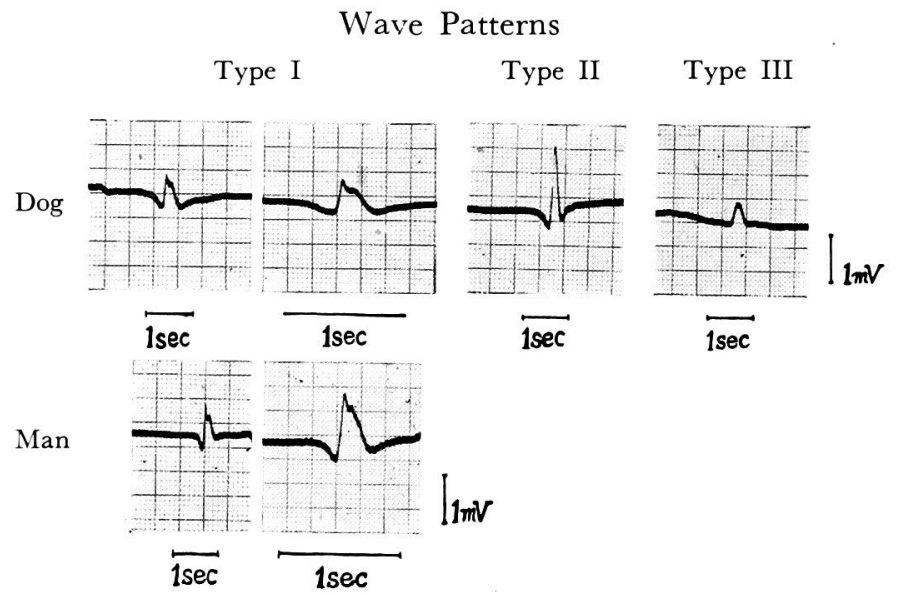

Fig. 4. For recording action potentials iron electrodes, Poly-Viso having time constant of $2.5 \mathrm{sec}$. and heat-writing recorder were used.

recorder. Some minute differences are found between the wave-patterns shown in Fig. 3 and Fig. 4, but these differences cannot be deemed essentially significant.

The frequency of appearance of the three types classified above was as follows. Among the 42 dogs tested, Type I occured in 35 tests, Type II in 28 tests and Type III in 17 tests, while among the 12 human subjects, Type I 
TABLE I

Frequency of Appearance of the Three Types

\begin{tabular}{l|c|c|c|c}
\hline & No. of cases & Type I & Type II & Type III \\
\hline Dogs & 42 & 35 & 28 & 17 \\
Men & 12 & 8 & 8 & 2
\end{tabular}

came forth in 8 tests, Type II also in 8 tests and Type III in only 2 tests. The frequency of Type III was thus low in dog as well as in man (Table I).

Such wave patterns are stable, for the same wave pattern would persist for 1-3 hours or even longer, as far as the electrical contact between the electrodes and the ureter remains unchanged. When, however, these conditions were changed, we found in many cases that the wave pattern changed over to another type.

When the uretral peristalsis was powerful, Type I appeared most frequently, Type II coming next and Type III being least frequenty, but when the peristaltic movement was weak, Type III was encountered most frequently, Types I and II being far less frequent. Besides, we encountered Type III very often when the room temperature fell below $10^{\circ} \mathrm{C}$, but Types I and II came forth more frequently when the temperature stood between $17^{\circ}$ and $25^{\circ} \mathrm{C}$.

Discharge intervals. The intervals of the electric discharges showed individual differences and also varied with the lapse of time in the same individual as shown in Fig. 5, we found 3 general types here too, namely, the cases with an approximately constant interval between the successive discharges, the cases showing cyclic changes consisting of two or three regualar discharges followed by a pause and the cases where the discharges occurred suite irregularly. Such interval patterns, however, were not constant per cases, for the discharges tended to occur at regular intervals at the initial stage of recording but tended to become interrupted by pauses or irregular in appearance with the lapse of time in many cases. The intervals tended to be equal and regular when the peristaltic movement of the ureter seemed strong, but irregular when only weak peristalsis was observed.

In the human subjects tested, the patterns of discharge intervals were nearly similar to those in the experimental animals, but irregularity in the intervals was more frequent among the former.

Measurements of discharge intervals, amplitude and duration. As shown in Fig. 6 , a discharge interval was measured by the number of seconds elapsed between two consecutive positive preceding deflections, $a$ and $a^{\prime}$ the amplitude $\mathrm{H}(\mathrm{mV})$ by the vertical distance from $a$ to the apex of the major negative discharge, $b$, and the duration $\mathrm{F}$ (sec.) of the potential by the 

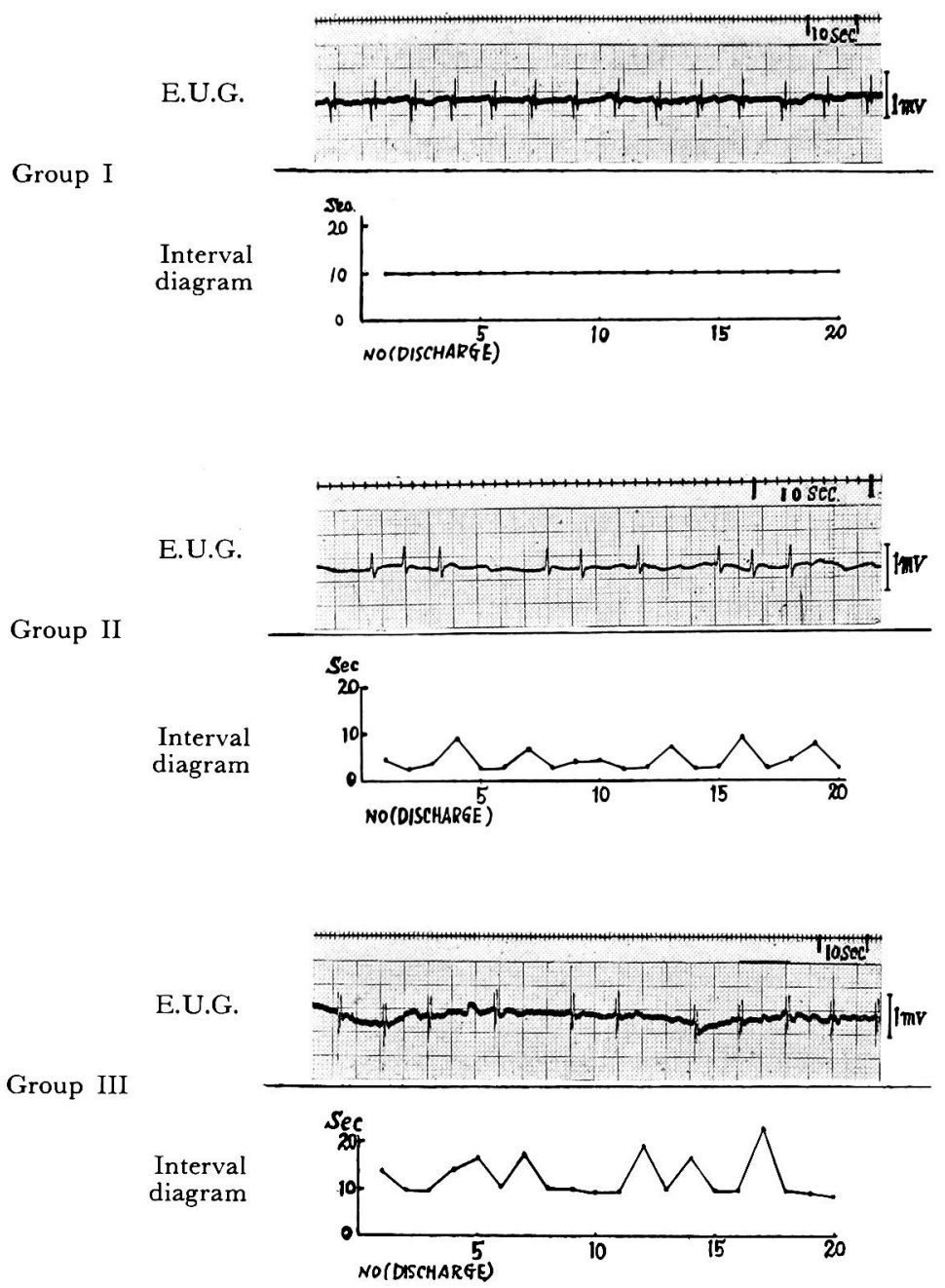

Fig. 5. Variation of discharge intervals in dogs. We found three general types. Group I, regular and constant intervals. Group II is showing cyclic change consisting of two or three discharges followed by a pause. Group III is showing quite irregular intervals.

time from $a$ to the apex of the positive after-potential, $d$.

The measured values of the discharge intervals, including the irregular intervals described above, ranged between 1 and 20 seconds in dogs and between 5 and 40 scconds in human subjects, the dogs in general showing shorter intervals (Fig. 7).

The amplitude of the discharges ranged between 0.5 and $1.5 \mathrm{mV}$ in dogs and between $0.3-1.7 \mathrm{mV}$ in men, the human and the canine subjects 
showing very small difference in this respect. The amplitude did not show any tendency to rise or fall with the lapse of time, in any test with the same ureter under the same test conditions (Fig. 7).

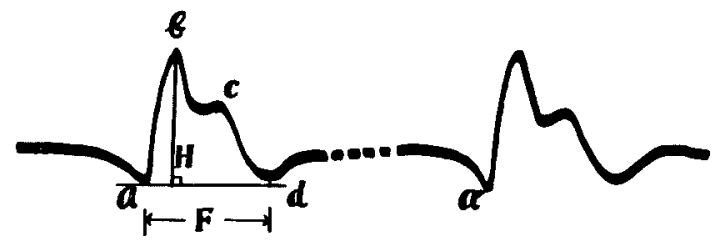

Fig. 6. Measurements of discharge interval, amplitude and duration. $a$, positive preceding deflection ; $b$, major negative deflection ; $c$, minor negative deflection ; $d$, positive after potential. $a a^{\prime}$, discharge interval (sec.) $\mathbf{H}$, amplitude $(\mathrm{mV}) ; \mathrm{F}$, duration (sec.).

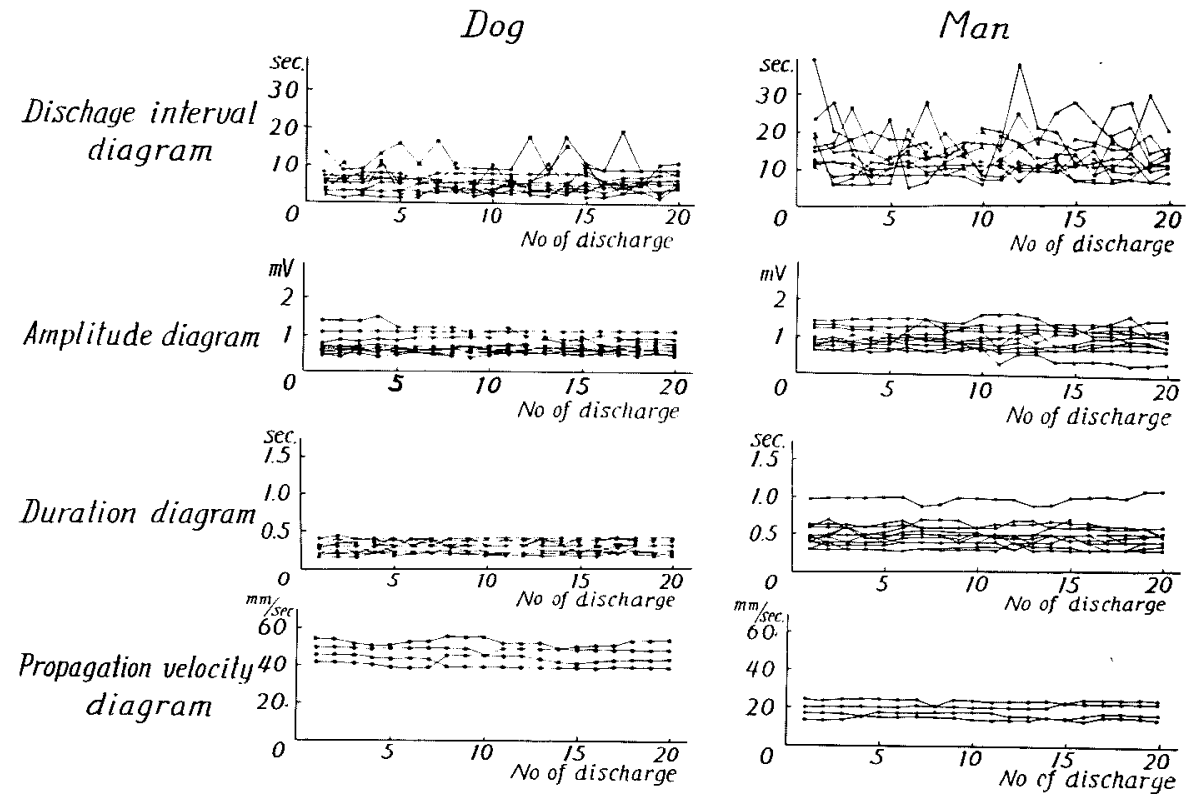

Fig. 7. Each curves in diagrams show progress of individual subject in dogs and men.

The duration of a discharge cycle changes in compliance with the change in wave patterns. In general, the duration ranged between 0.2 and $0.4 \mathrm{sec}$. in dog and between 0.3 and $1.1 \mathrm{sec}$. in man. The values were found usually smaller in the dogs than in the human subjects. As to the duration little temporary variations occurred over a very long period of recording (Fig. 7).

Measurements of propagation velocity. With bipolar leading we can 


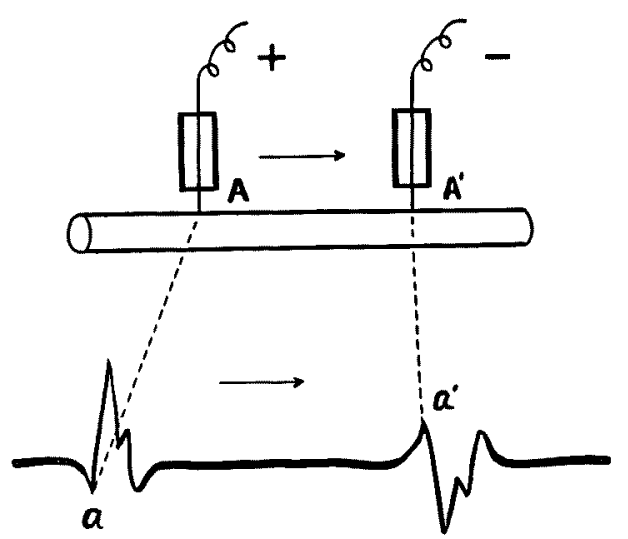

Fig. 8. Measurement of propagation velocity. $\mathrm{V}$ is propagation velocity ( $\mathrm{mm} / \mathrm{sec}$ ). AA', distance between electrodes (bipolar) (mm). a, preceding positive deflection at $\mathrm{A}$. $a^{\prime}$, preceding positive deflection at $\mathrm{A}^{\prime}$. $\mathrm{S}$, displacement speed of the recording paper in $\mathrm{mm} / \mathrm{sec}$.

measure the velocity of propagation. In Fig. 7, the proximallead is denoted by $\mathrm{A}$, and the distal by $\mathrm{A}^{\prime}$. Denote the preceding positive deflection at $\mathrm{A}$ with $a$ and that at $\mathrm{A}^{\prime}$ with $a^{\prime}$, then the propagation velocity $\mathrm{V}$ is given by the following formula:

$$
\mathrm{V}=\frac{\mathrm{AA}^{\prime} \times \mathrm{S}}{a a^{\prime}}
$$

Where $\mathrm{AA}^{\prime}, a a^{\prime}$ and $\mathrm{S}$ represent the distance between $\mathrm{A}$ and $\mathrm{A}^{\prime}$ on the ureter, the distance from $a$ to $a^{\prime}$ on the record, and the distance travelled by the recording paper per second respectively.

The measured values of propagation velocity were $38-56 \mathrm{~mm}$. $/ \mathrm{sec}$. in the dogs and 14-26 mm/sec, in the human subjects, the former showing a perceptibly higher velocity, but the change with time during any test of the same ureter, if any, was only very small (Fig. 7).

EUGs simultaneously recorded from ureters on both sides. With 10 dogs and 5 human patients as subjects, we made bipolar simultaneous recording of action potentials from both ureters of the same individuals and obtained such records as shown in Fig. 9. The wave forms of both potentials were similar in some cases but quite dissimilar in the others. The amplitude, the duration and the propagation velocity were always different in the right and left ureters, the intervals of discharges were also dissimilar, and we could not establish any antagonism between their potentials either.

\section{Summary AND Discussion}

The authors recorded spontaneous action potentials of the ureters 


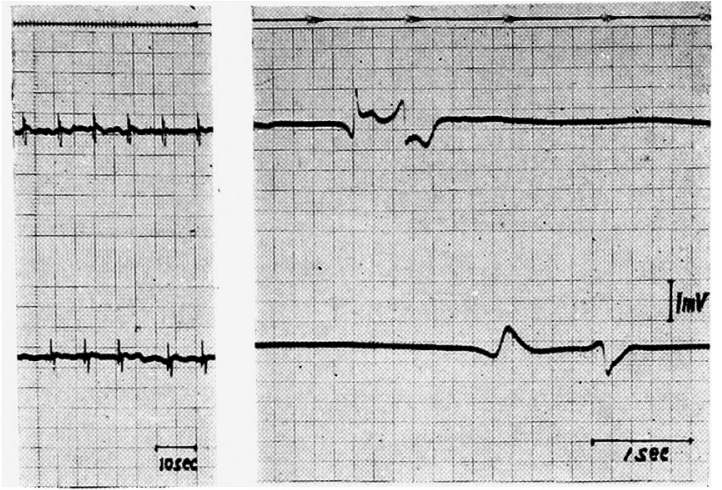

Fig. 9. EUGs simultaneously recorded from ureters on both sides of the same individual. Above, distance between electrodes is $25 \mathrm{~mm}$. Below, distance between electrodes is $37 \mathrm{~mm}$.

of dogs and human subjects and distinguished 3 different types of wave patterns. Our type I was found to coincide with that recorded by Butcher ${ }^{11}$ from human subjects, by Sleator ${ }^{10}$ ) from dogs and by Niu" from rats and cats, and our type II with that recorded by Orbeli and Brücke ${ }^{11}$ and $\mathrm{Aiba}^{3)}$ from dogs, by $\mathrm{Yano}^{5)}$ from pigs, by $\mathrm{Niu}^{7)}$ from rats and by $\mathrm{Obama}^{81}$ and $\mathrm{Kudo}^{9}$ ) from cats. The slow curve recorded by Corey ${ }^{12)}$ from human ureters was of complicated rorm, but devoid of spiky elements. This is probably because it represents a mass response recorded with a large ring electrode affixed to a catheter, and it was another characteristic of this record that the phase reversal as found in usual records was not clear in his records obtained with bipolar leading. The wave forms of the continuous uretral discharges obtained by Baker ${ }^{13)}$ with a subcutaneous injection needle (No. 28) are different from ours in that they were triphasic, but showed considerable mutual dissimilarity.

As has been mentioned above, we succeeded in obtaining potentials of wholly identical wave form over a period as long as 3 hours, and this must be evidence that potentials provide a serviceable means in studying the function of the ureter.

Butcher has found uretral discharges coming forth in patterns of equal intervals or of groups, but we could also detect some cases of irregular occurrences.

In our present study, we obtained the measured values of discharge intervals of 1-20 sec. in dogs and of 5-40 sec. in men, of amplitudes of 0.5$1.5 \mathrm{mV}$ in dogs and $0.3-1.7 \mathrm{mV}$ in men, of duration of $0.2-0.4 \mathrm{sec}$. in dogs and $0.3-1.1 \mathrm{sec}$. in men and of propagation velocity, of $38-56 \mathrm{~mm} / \mathrm{sec}$ in dogs and $14-26 \mathrm{~mm} / \mathrm{sec}$ in men, values approximately in agreement with the values obtained by Butcher ${ }^{11)}$ with human, by Sleator ${ }^{10)}$ with canine 
and by $\mathrm{Niu}^{6 / 7)}$ with ratty and feline ureters.

\section{Conclusion}

The authors recorded the spontaneous action potentials of the ureters exposed under laparotomy of 12 human subjects with intestinal diseases but diagnosed as healthy in their urinary tract and of 42 normal adult dogs over a long period of 1-3 hours, to examine the properties of such potentials and arrived at the following conclusions.

1. The wave patterns were similar in dog and in man. The typical action potential consisted of four deflections, namely a preceding positive deflection, a major negative deflection accompanied by a minor one and a positive after-potential. This type was called Type I. The wave pattern lacking the minor negative deflection was designated Type II, the pattern apparently consisting of a main negative deflection alone was named Type III. All these potentials seemed to arise in the normal state of the ureter.

2. Discharge of potentials occurred either at regular and constant intervals, or in groups, or quite irregularly.

3. The intervals of discharge ranged between 1 and 20 sec. in dog and between 5 and $40 \mathrm{sec}$. in man, their amplitude between 0.5 and 1.5 $\mathrm{mV}$ in $\operatorname{dog}$ and between $0.3-1.7 \mathrm{mV}$ in man, their duration between 0.2 and $0.4 \mathrm{sec}$. in dog and 0.3 and $1.1 \mathrm{sec}$. in man and the propagation velocity between $38-56 \mathrm{~mm} / \mathrm{sec}$ in $\mathrm{dog}$ and $14-26 \mathrm{~mm} / \mathrm{sec}$ in man. Both in man and dog, the discharge intervals changed only slightly with time, but almost no temporary change could be seen with other values such as maplitude, duration, etc. of such a change.

4. Simultaneous recording of the action potentials of ureters of both sides revealed no close correlation between the EUGs of both sides.

\section{References}

1) Orbeli \& Brücke, Pflügers Arch., 1910, 133, 341.

2) Hasama, ihid., 1933, 232, 236.

3) Aiba, Nisshin Igaku (Jap.), 1934, 23, 965.

4) Bozler, Amer. J. Physiol, 1942, 136, 553.

5) Yano, Tohoku J. Exp. Med., 1941, 41, 13.

6) Niu, Nippon Seirigaku Zassi (Jap.), 19.50, 12, 377.

7) Niu, ibid., 1951, 13, 6.

8) Obama, Denki Seirigaku Kenkyu (Jap.), 1955, 7, 59.

9) Kudo, ibid, 1955, 8, 67.

10) Sleator \& Butcher, J. Physiol., 1955, 180, 261.

11) Butcher \& Sleator, J. Urol., 1954, 73, 970.

12) Corey, Fite \& Samuel, ibid., 1956, 75, 244.

13) Baker \& Huffer, ibid., 1953, 70, 874. 